\title{
Exploring the Jet/Accretion Flow Relationship in Low Disk Luminosity Sources*
}

\author{
Sera Markoff \\ MIT, Center for Space Research, Cambridge, MA 02139, USA
}

\begin{abstract}
Astrophysical jets seem to gain strength disproportionate to the power of their associated accretion flow, making low-luminosity sources ideal targets for studies of the role of outflows. Radio/X-ray correlations have supported the case for a strong relationship between the jets and the hard X-ray emitting regions, and here we explore the strongest scenario where the base of the jets subsumes the role of the corona. The properties of coronae, as inferred from spectral models, are very similar to what is empirically required at jet bases assuming conservation laws hold. We present a few preliminary fits to simultaneous radio and X-ray data sets from our GX 339-4 and Cyg X-1 campaigns. The fits are performed in detector space, and include a jet plus thermal disk continuum model, with added Gaussian line and non-relativistic reflection features similar to the approach of other X-ray models. We find that we can fit the entire radio through X-ray spectrum quite well, with any deviation occurring in the line/reflection region. The results suggest that a jet/corona unification can provide a reasonable description of the data. Future work will benefit from a more complex approach to the disk feedback features.
\end{abstract}

\section{Introduction}

Relativistic outflows have been considered part of the big picture for active galactic nuclei (AGN) almost since their discovery, simply because their scales are so large compared to the host galaxy in radio frequencies. The confirmation that jets also exist in smaller accreting compact objects, such as X-ray binaries (XRBs), was not fully established until the late 1970s (SS433; [31]). However, XRB jets continued to be treated as distinct components until fairly recently, when correlations between the radio and X-ray luminosities were discovered in the hard state of GX 339-4 $[15,5,6]$. This correlation is now thought to be fundamental to accreting black holes $[12,26,8]$. The realization that the correlation scales with mass has provided a new method of comparison between AGN and XRBs, and for the exploration of the spectral roles of the individual components.

Despite this progress, the details of the inflow/outflow interface are still a matter of significant debate. The nature of the connection is important for models of jet formation, and in general our understanding of the effects of strong gravitational fields on magneto-hydrodynamical

* NSF Astronomy \& Astrophysics Postdoctoral Fellow

(c) 2018 Kluwer Academic Publishers. Printed in the Netherlands. 
plasmas. An important way to approach this problem is to create physical models which consider the system holistically, by both predicting the broadband spectra at the same time as addressing fine features specific to the X-ray band. Since the radio emission in these sources is accepted to come from jets we have a solid anchor for models which probe how jets relate to the X-ray emission.

Low disk luminosity systems are characterized by their sub-Eddington accretion rates and steady compact jets. They are advantageous sources for this study because one can avoid the complications of high accretion rates as seen in, e.g., AGN, especially near the base of the jets where the physics is most in question. Furthermore, because the evidence is increasing that jets dominate the power at low luminosities (e.g., $[10,19])$ we can better hope to isolate the roles of jets in these sources.

\section{Model Background}

\subsection{JET MODEL DEVELOPMENT}

We first explored scaling jet models for Sgr A*, whose quiescent and flared emission can be explained via synchrotron radiation in the radio frequencies, and synchrotron and/or synchrotron self-Compton (SSC) in the X-ray band $[9,21]$. This model scales with the central mass and accretion power, and thus in principle can be applied to XRBs as well. XTE J1118+480 was the ideal test source, with its unprecedented highquality broadband, quasi-simultaneous data ([16, 25], and refs. therein). We had originally set out to model the radio spectrum via synchrotron emission, but it was immediately apparent that the scaling resulted in the optically thick-to-thin break occurring in the infrared/optical bands. More importantly, we found that if a power law distribution of energetic particles exists further out in the jet, it is in fact quite difficult to suppress the optically thin synchrotron radiation from extending out into the X-ray band [20]. An accelerated power law distribution of particles is motivated by observations of optically thin emission during radio outbursts in XRBs (e.g., [11]) and AGN (e.g., [24]). The jet synchrotron-dominated model presented in [20] gives a good description of the broad spectral features of XTE J1118+480, with the $\sim 100 \mathrm{keV}$ cutoff following from acceleration saturated by synchrotron cooling. Thermal emission from the inner edge of an accretion disk was included in the calculation both as a weak direct component and as seed photons for inverse Compton scattering, but was not the dominant emission component.

We next considered this model for 13 (quasi)-simultaneous radio/Xray (and sometimes IR) data sets for GX 339-4 [6]. We found again 
that jet synchrotron could explain the broadband continuum spectra of all the observations, mainly by just varying the power input into the jet [22]. We showed in this paper that jet synchrotron emission analytically predicts the slope of the radio/X-ray correlation as a consequence of its scaling with power.

In summary, these simple synchrotron-dominated jet models significantly developed our understanding of the radio/X-ray correlations and scaling. However, they did not attempt to address the fine features in the X-ray spectrum, which are harbingers of interactions with cooler accretion disk material.

\subsection{JET/CORONA RELATIONSHIP}

Accounting for the hard state X-ray power law spectrum, in combination with the line emission/reflection features attributed to interaction with the accretion disk, led to the development of the corona/disk model (e.g., $[14,13])$. The corona is inferred from X-ray signatures to have several properties, such as quasi-thermal electrons in a reasonably compact geometry. The fraction of reflected hard X-ray emission is often low in the hard state, and this fact is sometimes problematic for corona models [7]. Various mechanisms have been proposed to decrease the fraction of reflected X-rays, including patchy coronae [32], high disk ionization $[29,27,1]$ and beaming of the coronae away from the disk with mildly relativistic velocities $[2,18]$. This latter approach seems extremely close in principle to the characteristics of the base of a jet.

Unlike corona jets are observed explicitly: the synchrotron emission tells us that there is a population of accelerated leptons quite far out in the jet. If one believes that there is rough conservation of properties along the jet, then tracing back to the base requires hotter leptons in even denser populations threaded by even stronger magnetic fields. At the same time, magnetohydrodynamical simulations of the inner disk region do not show anything like a stable sphere/disk geometry, but rather a region threaded with fields which naturally leads to outflowing plasma (corona?) (e.g., [33]). This beaming will reduce the relevance of the already weak thermal disk photons with respect to locally created photons and lead to an altogether different picture than the static case. We think it is worth exploring the most extreme, and thus easiest to test, case scenario: whether the base of the jets can "subsume" the role of the corona. This is a critical step in the process of disentangling their actual relationship, since if the approach fails, where and how it fails will provide clues as to the nature of the corona as a distinct component. If it does not fail, it may help point the way towards a better understanding of jet formation. 

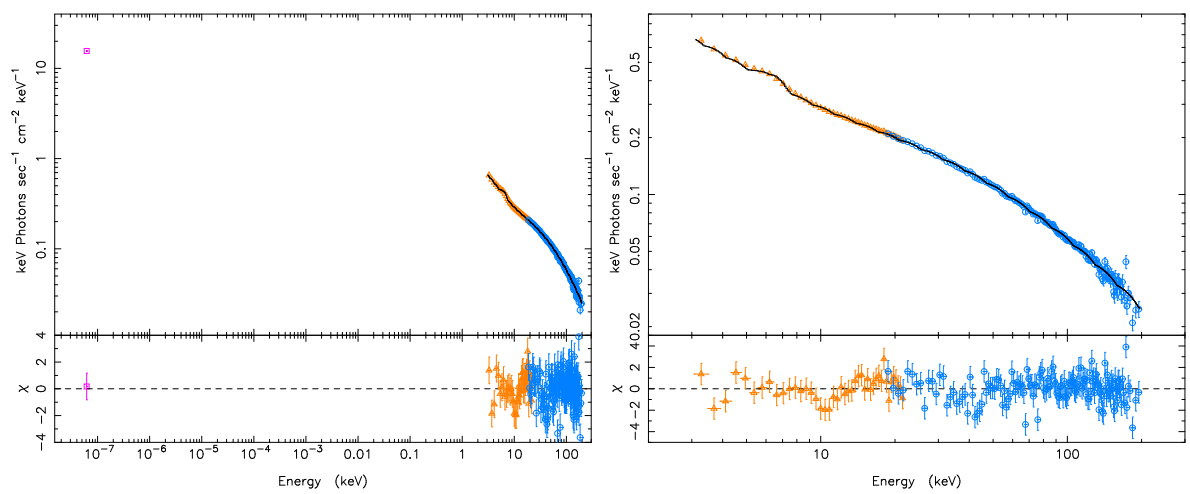

Figure 1. The best fit so far of our three Cyg X-1 simultaneous radio/X-ray observations which span its typical spectral variations in the hard state, $\chi^{2}=208.4 / 183=1.14$. The figures show the unfolded fit plus residuals with an absorbed jet model (including a thermal accretion disk component) plus Gaussian line, convolved with non-relativistic reflection. a) The entire broadband fit, including the radio data point, b) close-up on the X-ray bands. X-ray data are from RXTE PCA \& HEXTE, radio from the Ryle Telescope.

\subsection{Model Background}

A more detailed explanation can be found in other papers $([20,22]$; Markoff, Nowak \& Wilms in prep.) but the basic model is a freely expanding jet which has a velocity gradient due to acceleration along its axis. Plasma enters the jet at the base, and the radiation is dominated by quasi-thermal leptons which cool radiatively and adiabatically as the jet expands. Further out in the jet the particles encounter an acceleration region where some of them are accelerated into a power law tail. The particles radiate along the entire jet via synchrotron, synchrotron self-Compton (SSC) and external Compton (EC) radiation. The EC only contributes very close to the base of the jet, where it can be comparable to the SSC, both of which contribute to the hard X-rays. Synchrotron emission from $\sim 100 r_{\mathrm{g}}$ dominates in the radio/IR bands, turning over to contribute in the X-ray band.

Our currently favored model is a direct result of our work in [23], where we calculated the reflection from typical jet models based on GX 339-4 spectra. We found this a very useful probe of the geometry of jet emitting regions, which helped define our latest models. If the acceleration region really exists further out in the jet, as we have found for several fits, then the weakly beamed synchrotron radiation cannot result in more than a few percent reflection (assuming perpendicular geometry and a flat disk). SSC near the base of the jets, however, can easily give $\lesssim 20 \%$ in the simplest case. 

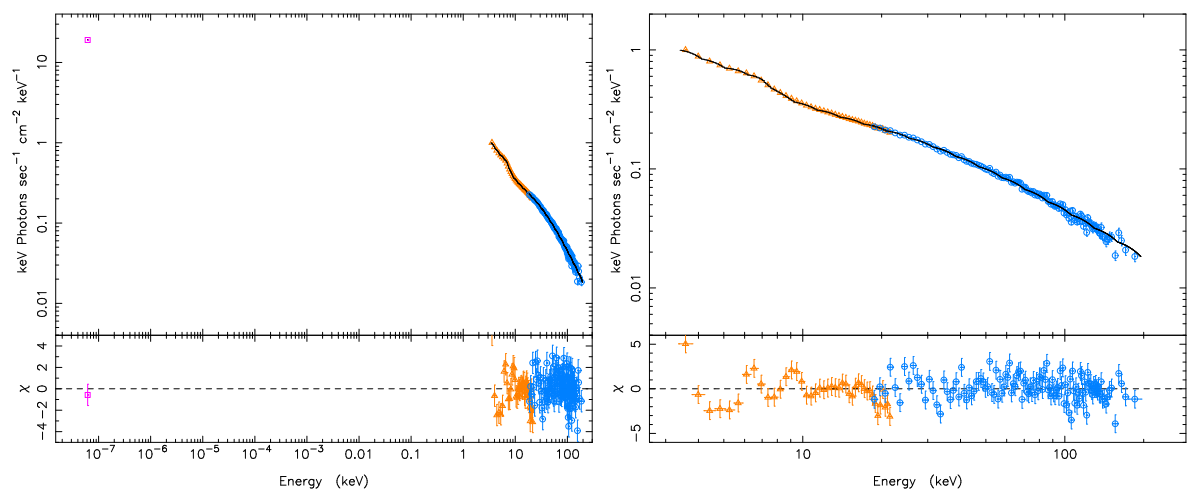

Figure 2. The worst fit so far of our three Cyg X-1 simultaneous radio/X-ray observations which span its typical spectral variations in the hard state, $\chi^{2}=278 / 162=1.72$. Otherwise same format as Fig. 1 .

In order to address the fine features of the X-ray spectrum in a statistical manner, we needed to import this model into X-ray data analysis software. Currently it is running in XSPEC and ISIS, and the figures here were made with the latter program. In comparison to models which focus exclusively on the X-ray frequencies, however, we are also importing and fitting the simultaneous radio data with our model. Within the program, we added a single Gaussian line to our continuum model, and allowed it to vary between $6-7 \mathrm{keV}$. We then convolved the entire spectrum with a non-relativistic reflection model derived from the Greens functions of [17]. Because of the complexities of directly calculating the interaction of jet photons with the accretion disk (see [23]), we could not self-consistently include these features but include the strength of the reflection hump as a free parameter. This is similar to the general approach chosen by pure Comptonization models (e.g., $[3,28])$. The full calculation of these features requires a Monte Carlo approach, which we will consider elsewhere.

\section{Results}

Here we present the best and worst fits so far from three data sets of Cyg $\mathrm{X}-1$ which span its typical range in hard state spectral characteristics (Figs.1 \& 2, respectively). GX 339-4 varies mainly in luminosity rather than spectral shape, and so we present just one typical fit in Fig. 3. The data come from the Rossi X-ray Timing Explorer (RXTE), using both the Proportional Counter Array (PCA) and the High Energy X-ray Timing Experiment (HEXTE; [30]), extracted with the newest release 

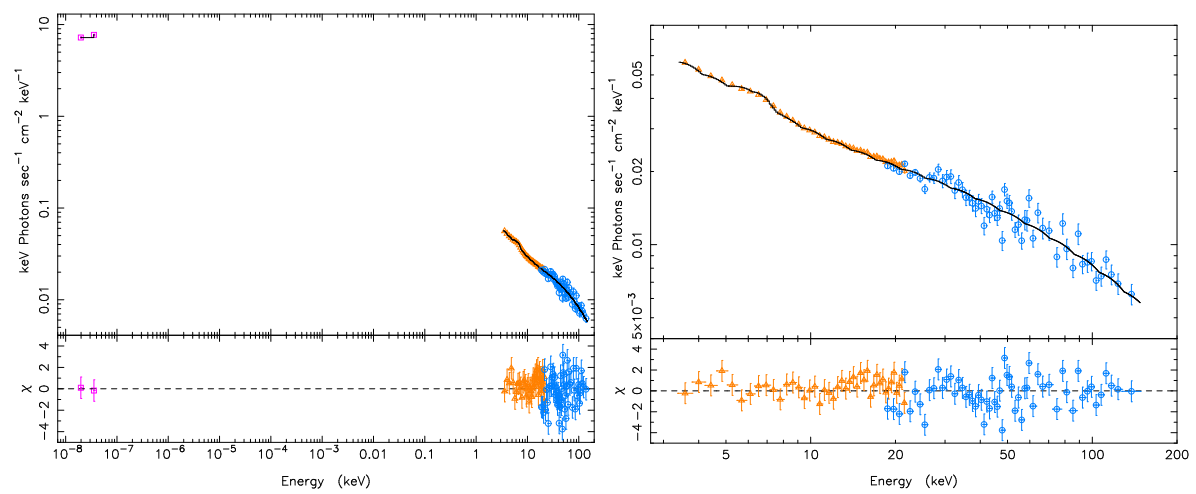

Figure 3. Typical fit from three simultaneous radio/X-ray observations of GX 339-4 which span its luminosity variations in the hard state, $\chi^{2}=138.3 / 96=1.44$. Otherwise same format as Figs. $1 \& 2$.

of the RXTE software, HEASOFT 5.3.1. The radio data come from the ATCA (GX 339-4) and Ryle (Cyg X-1) instruments.

\section{Discussion}

The fits presented here are comparable to fits of the X-ray data alone made with broken power law models (Nowak, this volume), and thermal Comptonization models (e.g. EQPAIR, [4]). Unfortunately, the multicomponent jet model currently runs significantly slower than singlecomponent corona models, which means we are not able to explore the same amount of parameter space in a reasonable amount of time (yet). However, it is important to realize that since we are including the radio self-consistently, we have a more holistic approach to a system we know is coupled. The main thing to note is that the cutoff region is not where the fit has its limitations, but rather in the line/reflection regime. This is most likely due to the lack of time for finding slightly better parameters, as well as the use of simple, non-relativistic, non-ionized models for the disk component. Secondly, the reflection fractions required for these fits are lower than those inferred for thermal Comptonization models ( $\sim 5-10 \%$ compared to $15-20 \%)$. This is mainly due to differences in the shape of the continuum for the two types of models, because in the jet model the SSC component already lends its curvature to the hard X-rays, reducing the need for strong reflection. At the same time, these lower reflection fractions are entirely consistent with our estimations of reflection from jet models, and thus demonstrates that the phenomenology of reflection is very dependent on assumptions of the impinging continuum. 
So far it seems a model where the jet takes over the role of the corona, or includes it, is statistically feasible. The next step is to consider tighter constraints on the shape of the spectrum at the highest energies near the cutoff, which may break some of the degeneracy. The upcoming mission ASTRO-E2 will hopefully provide useful data for these tests. Similarly, timing studies will be valuable for further constraining the location of the emitting regions.

\section{Acknowledgements}

Some parts of this talk evolved into Markoff, Nowak and Wilms (in prep., 2005), and so thanks go to my collaborators Michael Nowak and Jörn Wilms. I also would like to thank Heino Falcke, Stéphane Corbel and Rob Fender for their collaboration in the earlier development of this work, as well as Guy Pooley for his persistent monitoring of Cyg X-1. Support was provided by an NSF Astronomy \& Astrophysics postdoctoral fellowship under NSF Award AST-0201597, as well as NSF grant INT-0233441.

\section{References}

1. Ballantyne, D. R., Iwasawa, K., \& Fabian, A. C. 2001, MNRAS, 323, 506

2. Beloborodov, A. M. 1999, ApJ, 510, L123

3. Coppi, P. S. 1992, MNRAS, 258, 657

4. Coppi, P. 1999, PASP Conference Series, 161, 375

5. Corbel, S., Fender, R. P., Tzioumis, A. K., Nowak, M., McIntyre, V., Durouchoux, P., \& Sood, R. 2000, A\&A, 359, 251

6. Corbel, S., Nowak, M., Fender, R. P., Tzioumis, A. K., \& Markoff, S. 2003, A\&A, 400, 1007

7. Dove, J. B., Wilms, J., Maisack, M. G., \& Begelman, M. C. 1997, ApJ, 487, 759

8. Falcke, H., Körding, E., \& Markoff, S. 2004, A\&A, 414, 895

9. Falcke, H. \& Markoff, S. 2000, A\&A, 362, 113

10. Fender, R. P., Gallo, E., \& Jonker, P. G. 2003, MNRAS, 343, L99

11. Fender, R. P. \& Kuulkers, E. 2001, MNRAS, 324, 923

12. Gallo, E., Fender, R. P., \& Pooley, G. G. 2003, MNRAS, 344, 60

13. George, I. M. \& Fabian, A. C. 1991, MNRAS, 249, 352

14. Haardt, F. \& Maraschi, L. 1991, ApJ, 380, L51

15. Hannikainen, D. C., Hunstead, R. W., Campbell-Wilson, D., \& Sood, R. K. 1998, A\&A, 337, 460

16. Hynes, R. I., Mauche, C. W., Haswell, C. A., Shrader, C. R., Cui, W., \& Chaty, S. 2000, ApJ, 539, L37

17. Magdziarz, P. \& Zdziarski, A. A. 1995, MNRAS, 273, 837

18. Malzac, J., Beloborodov, A. M., \& Poutanen, J. 2001, MNRAS, 326, 417

19. Malzac, J., Merloni, A., \& Fabian, A. C. 2004, MNRAS, 351, 253 
20. Markoff, S., Falcke, H., \& Fender, R. 2001, A\&A, 372, L25

21. Markoff, S., Falcke, H., Yuan, F., \& Biermann, P. L. 2001b, A\&A, 379, L13

22. Markoff, S., Nowak, M., Corbel, S., Fender, R., \& Falcke, H. 2003, A\&A, 397, 645

23. Markoff, S. \& Nowak, M. A. 2004, ApJ, 609, 972

24. Marscher, A. P. \& Gear, W. K. 1985, ApJ, 298, 114

25. McClintock, J. E., Haswell, C. A., Garcia, M. R., Drake, J. J., Hynes, R. I., Marshall, H. L., Muno, M. P., Chaty, S., Garnavich, P. M., Groot, P. J., Lewin, W. H. G., Mauche, C. W., Miller, J. M., Pooley, G. G., Shrader, C. R., \& Vrtilek, S. D. 2001, ApJ, 555, 477

26. Merloni, A., Heinz, S., \& di Matteo, T. 2003, MNRAS, 345, 1057

27. Nayakshin, S. 2000, ApJ, 534, 718

28. Poutanen, J. 1998, in Theory of Black Hole Accretion Disks, Cambridge University Press, 100

29. Ross, R. R., Fabian, A. C., \& Young, A. 1999, MNRAS, 306, 462

30. Rothschild, R. E., Blanco, P. R., Gruber, D. E., Heindl, W. A., MacDonald, D. R., Marsden, D. C., Pelling, M. R., Wayne, L. R., \& Hink, P. L. 1998, ApJ, 496, 538

31. Spencer, R. E. 1979, Nat., 282, 483

32. Stern, B. E., Poutanen, J., Svensson, R., Sikora, M., \& Begelman, M. C. 1995, ApJ, L13

33. Stone, J. M. \& Pringle, J. E. 2001, MNRAS, 322, 461 